\title{
Disease Response Original Result
}

National Cancer Institute

\section{Source}

National Cancer Institute. Disease Response Original Result. NCI Thesaurus. Code C117397.

The outcome of the disease response assessment as originally received or collected. 